\title{
MAPK4 deletion enhances radiation effects and triggers synergistic lethality with simultaneous PARP1 inhibition in cervical cancer
}

Shuzhen Tian ${ }^{1 *}$, Lili Loư ${ }^{2}$, Mengyuan Tian ${ }^{3}$, Guangping Lu' ${ }^{4}$ Jianghua Tian ${ }^{5}$ and Xi Chen ${ }^{6}$

\begin{abstract}
Background: Cervical cancer is one of the most common cancers among females worldwide and advanced patients have extremely poor prognosis. However, adverse reactions and accumulating resistance to radiation therapy require further investigation.

Methods: The expression levels of mitogen-activated protein kinase 4 (MAPK4) mRNA were analyzed by real-time PCR and its association with overall survival was analyzed using Kaplan-Mier method. Colony formation,

immunofluorescence and western blotting were used to examine the effects of MAPK4 knockout or over-expression on cervical cancer cells after radiation treatment. Drug-sensitivity of cervical cancer cells to PARP1 inhibitors, olaparib or veliparib, was analyzed by CCK-8 cell viability assays, and the 50\% inhibitory concentration (IC50) was quantified using GraphPad Prism. The functional effects of MAPK4 knockout on the sensitivity of cervical cancer to radiation treatment and PARP1 inhibitors were further examined using xenograft tumor mouse models in vivo.

Results: Cervical cancer patients with high MAPK4 mRNA expression have lower survival rate. After radiation treatment, the colony number of MAPK4 knockout cells was markedly reduced, and the markers for DNA double-chain breakage were significantly up-regulated. In addition, MAPK4 knockout reduced protein kinase B (AKT) phosphorylation, whereas its over-expression resulted in opposite effects. In MAPK4 KO cells with irradiation treatment, inhibition of AKT phosphorylation promoted DNA double-chain breakage. Constitutive activation of AKT (CA-AKT) increased the levels of phosphorylated-AKT ( $p$-AKT), and DNA repair-related proteins, phosphorylated-DNA-dependent protein kinase ( $p$-DNAPK) and RAD51 recombinase (RAD51). Furthermore, MAPK4 knockout was found to affect the sensitivity of cervical cancer cells to poly ADP-ribose polymerase 1 (PARP1) inhibitors by activating the phosphorylation of AKT. Moreover, in vivo results demonstrated that MAPK4 knockout enhanced the sensitivity of cervical cancer to radiation and PARP1 inhibitors in mouse xenograft models.

(Continued on next page)
\end{abstract}

\footnotetext{
*Correspondence: ShuzhenTianfjk@163.com

'Department of Gynecology, Affiliated Cancer Hospital of Zhengzhou

University, Henan Procincical Cancer Hospital, No. 127 Dongming Avenue,

Zhengzhou City 450009, Henan Province, China

Full list of author information is available at the end of the article
}

\section{$\triangle B M C$}

(c) The Author(s). 2020 Open Access This article is licensed under a Creative Commons Attribution 4.0 International License, which permits use, sharing, adaptation, distribution and reproduction in any medium or format, as long as you give appropriate credit to the original author(s) and the source, provide a link to the Creative Commons licence, and indicate if changes were made. The images or other third party material in this article are included in the article's Creative Commons licence, unless indicated otherwise in a credit line to the material. If material is not included in the article's Creative Commons licence and your intended use is not permitted by statutory regulation or exceeds the permitted use, you will need to obtain permission directly from the copyright holder. To view a copy of this licence, visit http://creativecommons.org/licenses/by/4.0/. The Creative Commons Public Domain Dedication waiver (http://creativecommons.org/publicdomain/zero/1.0/) applies to the data made available in this article, unless otherwise stated in a credit line to the data. 
(Continued from previous page)

Conclusions: Collectively, our data suggest that combined application of MAPK4 knockout and PARP1 inhibition can be used as therapeutic strategy in radiation treatment for advanced cervical carcinoma.

Keywords: Cervical cancer, MAPK4, AKT phosphorylation, DNA double-chain breakage, Radiation sensitivity, PARP1 inhibitors

\section{Background}

With over half a million cases occurring annually worldwide, cervical cancer remains the second most common cancer and third leading cause of cancer-related death among females in developing countries [1-3]. Although human papillomavirus (HPV) vaccines are available and are effective in reducing the incidence and mortality, improved treatment for cervical cancer remains an urgent need [4]. In particular, patients with advanced cervical cancer have extremely poor prognosis and low survival rate [5]. According to National Comprehensive Cancer Network (NCCN) 2016, radiotherapy can be performed in all stages of patients [6]. However, increased dose of radiation inevitably leads to adverse reactions, and accumulating resistance to radiation therapy needs to be taken into account [7]. Therefore, questions remain as to which factors contribute to the radioresistance and poor outcomes of cervical cancer.

As a family of evolutionarily conserved enzymes, (mitogen-activated protein kinase) MAP kinases play a critical role in signaling cascades, which mediate a wide range of extracellular stimuli and result in different cellular responses, including cell proliferation, cell motility, cell cycle, cytokine biosynthesis, and chromatin remodeling and so on [8]. A total of 13 human MAP kinases have been identified, including ERK1/2 (MAPK3/1), P38 $\alpha / \beta / \gamma / \delta$ (MAPK14/11/ 12/13) and JNK1/2/3 (MAPK8/9/10) [9]. MAPK4, also known as ERK4, p63 MAPK, ERK3 $\beta$ and Prkm4, has been mapped to chromosome 18q12-21 following the identification of ERK1, ERK2 and ERK3 [10]. As an atypical MAPK, MAPK4 lacks TXY and APE motifs, which are highly conserved in other MAPKs. Instead, MAPK4 consists of a SEG sequence and SPR motif. Thus, MAPK4 can not be phosphorylated by the dual Ser/Thr and Tyr MAPK kinase (MAPKK) $[11,12]$. Transcriptomic profiling data provided by The Cancer Genome Atlas (TCGA) [13] show that MAPK4 expression is correlated with the survival rates in patients with lung cancer, bladder cancer and glioma. A recent report demonstrated that MAPK4 activates protein kinase B (AKT) in a phosphatidylinositol 3-hydroxykinase (PI3K)-independent manner and promotes cell proliferation and xenograft tumor growth [14]. After exposure to ionizing radiation, AKT phosphorylation plays a pivotal role in the stimulation of DNA-PKcs and recruitment of AKT1/ DNA-PKcs complex as an initiating step of DNA doublestrand break (DNA-DSB) repair [15]. A single gene may play different roles depending on the tissue context. In this study, the expression and function of MAPK4 were determined in cells of lung cancer, colon cancer and prostate cancer. Nevertheless, the functions of MAPK4 in radiation and its involvement in diseases, including cervical cancer, requires further investigation.

As an abundant nuclear protein implicated in DNA single-strand break (DNA-SSB) repair, PARP1 plays a vital role in catalyzing poly ADP-ribose formation and maintaining genome integrity [16]. Suppression of PARP1 leads to defective DNA-SSB repair and results in accumulation of DNA-DSB [17]. Given the regulatory roles of MAPK4/AKT and PARP1 in DNA-DSB and DNA-SSB repair, respectively, the possible synergistic effects between MAPK4 deletion and PARP1 inhibition requires further elucidation. Previous study showed that PARP1 hyperactivation leads to therapeutic resistance, and the therapeutic potential of PARP inhibition in combination with cisplatin has shown profound anti-cancer effect in cervical cancer [18]. In addition, high PARylation activity is correlated with sensitivity to olaparib in cervical cancer cells, and represents a biomarker for the identification of patients likely to benefit from PARP1 inhibition [19]. However, the synergistic role of PARP1 inhibition in combination with MAPK4 intervention for cervical cancer therapy is yet to be elucidated.

Here we report that high MAPK4 expression is associated with significantly decreased overall survival rate in cervical cancer patient. Cervical cancer cells were more sensitive to radiation treatment after MAPK4 knockout, as determined by colony formation, immunofluorescence and western blotting. Based on the results from the protein analysis, we found that MAPK4 specifically activated AKT phosphorylation and further affected DNA repair. In addition, MAPK4 knockout enhanced the sensitivity of cervical cancer cells to PARP1 inhibitors, olaparib and veliparib. The synergistic lethality of MAPK4 deletion in combination with PARP1 inhibition was further evaluated in a xenograft mouse model. Together, these results demonstrated that MAPK4 knockout alongside with PARP1 inhibition may improve the therapy of cervical cancer. 


\section{Methods}

\section{Patient tissue samples}

Paired cervical cancer tissues (tumor, $n=60$ ) and normal adjacent tissues (non-tumor, $n=60$ ) were obtained from the Henan Cancer Hospital. All patients were pathologically and clinically diagnosed as cervical cancer and received no chemotherapy or radiotherapy before section. The collection and experiments were performed under the permission of the Ethics Committee of Henan Cancer Hospital (Approval no.2016HC047). Written informed consents were obtained from all participants.

\section{Quantitative real-time PCR (qRT-PCR)}

First, total RNA was extracted from tissues and cells using the TRIzol reagent (TaKaRa, Otsu, Japan) according to the manufacturer's instructions. Total RNA was then converted into complementary DNA (cDNA) using a PrimerScript ${ }^{\mathrm{T} \mu}$ RT Reagent Kit (TaKaRa), following standard procedures. qRT-PCR was performed using a One-Step SYBR PrimeScript RT-PCR Kit (TaKaRa) on a 7500 Real-Time PCR System (Applied Biosystems, Lincoln Centre Drive Foster City, CA). $\beta$-Actin was used as a housekeeping gene to normalize the gene expression. The primer sequences for MAPK4 and RAD51 recombinase (RAD51) are listed in Table S1.

\section{Overall survival analysis}

After qRT-PCR analysis, mean MAPK4 mRNA level was calculated and used as a criterion to estimate the expression of MAPK4. If the MAPK4 mRNA level was higher than the mean value, the corresponding patient was identified as "MAPK4 high", whereas an MAPK4 mRNA level lower than the mean value was denoted as "MAPK4 low". After surgery combined with radiotherapy or postoperative radiotherapy, follow-up data was collected and the overall survival rate of cervical cancer patients in "MAPK4 high" and "MAPK4 low" group was analyzed using the Kaplan-Meier method.

\section{Cell culture}

In the present study, human cervical cancer cells, $\mathrm{SiHa}$ and caSki, were obtained from the ATCC cell lines (Manassas, USA). Cells were recently authenticated by STR profiling. Eagle's Minimum Essential Medium (EMEM) and RPMI-1640 medium were used to culture $\mathrm{SiHa}$ and caSki cells, respectively. Cells were cultured in medium supplied with $10 \%$ FBS (Thermo Fisher Scientific) in a humidified atmosphere with $5 \% \mathrm{CO}_{2}$ at $37^{\circ} \mathrm{C}$.

\section{Generation of MAPK4 knockout cell lines}

$\mathrm{SiHa}$ and caSki cells with MAPK4 deletion were established by CRISPR-Cas9 technique as previously reported [14]. Briefly, lentiviral packaging vectors pMD2.G and psPAX2 along with sgRNA-expressing vector, CRISPR- v2 vector, were transfected into НEK293T cells using the Lipo2000 transfection reagent (Thermo Fisher Scientific). The sgRNA sequence for MAPK4 used in study is ACTTCACTGTTCACTTCAGGGAG. Viruses were collected $72 \mathrm{~h}$ after transfection and infected $\mathrm{SiHa}$ and caSki cells. Then, MAPK4-deleted stable cell lines were selected by puromycin and were continuously monitored by western blotting to avoid potential reversal effects.

\section{Western blotting}

Western blotting was performed as previously reported [20]. Briefly, we collected protein lysates from cells and tissues using RIPA lysis buffer (Sigma-Aldrich, $20 \mathrm{mM}$ Tris $\mathrm{pH} 7.5,150 \mathrm{mM} \mathrm{NaCl}, 1 \mathrm{mM}$ PMSF, $10 \mathrm{mM} \beta$ glycerophosphate, $1 \%$ Triton X-100, $5 \mathrm{mM}$ EDTA, 0.2 $\mathrm{mM} \mathrm{Na} \mathrm{VO}_{4}, 2 \mu \mathrm{g} / \mathrm{mL}$ leupeptin, $2 \mu \mathrm{g} / \mathrm{mL}$ pepstatin A). Protein lysates were quantified by a BCA protein assay kit (Thermo Fisher Scientific) and loaded onto the sodium dodecyl sulfate-polyacrylamide gels (SDS-PAGE) for electrophoresis. Next, proteins were transferred onto a PVDF membrane. Membranes were blocked with 5\% BSA, and incubated with primary antibodies at $4{ }^{\circ} \mathrm{C}$ overnight. After that, HRP (horseradish peroxidase)-conjugated secondary antibodies were used to incubate the membrane. Immunoreactivity was determined by an immobilon ECL western HRP substrate and (Millipore, Billerica, MA, USA) and a chemiluminescence imaging system. $\beta$-actin protein level was used as a control. Antibodies used in this study are as follows: MAPK4, phosphorylatedDNA-dependent protein kinase (p-DNA-PK), RAD51, pAKT T308, p-AKT s473, AKT, AKT1, AKT2, p-AKT and phosphorylated histone $\mathrm{H} 2 \mathrm{AX}(\gamma \mathrm{H} 2 \mathrm{AX})$. Western blot bands were quantified by ImageJ and the quantification is shown in histogram. Antibody details are listed in Table S2.

\section{Irradiation and colony formation assay}

Irradiation was performed by X-rays at a dose rate of 5 Gy/min using a linear accelerator (PRIMUS-M, Siemens) for $2 \mathrm{~h}$. After radiation treatment, $\mathrm{SiHa}$ or caSki cells were primarily blended into top agar, and the mixture was then added onto base agar. After three weeks, colonies were stained with crystal violet and counted by dissection microscope (Nikon, Tokyo, Japan).

\section{Immunofluorescence}

Cells were seeded and treated with 5 Gy irradiation following cell adhesion. After fixation with $4 \%$ paraformaldehyde, $\mathrm{SiHa}$ or caSki cells were permeabilized in $0.1 \%$ Triton X-100. Then, cells were blocked with 5\% BSA and incubated with anti-53BP1 and anti- $\gamma \mathrm{H} 2 \mathrm{AX}$ primary antibodies overnight at $4{ }^{\circ} \mathrm{C}$. Cy5-conjugated and fluorescein isothiocyanate (FITC)-conjugated secondary antibodies were used. To visualize nuclear DNA, cell nuclei were stained with DAPI (4',6-diamidino-2-phenylindole, 
Sigma-Aldrich, St. Louis, MO, USA). Cells were observed and photographed using a confocal microscope (Nikon).

\section{Cell transfection and cell treatment}

$\mathrm{SiHa}$ or caSki cells were transfected with MAPK4 expressing plasmid (MAPK4), shRNA for MAPK4 (sh-MAPK), siRNA for AKT1 and AKT2 (si-AKT1, si-AKT2), plasmid with constitutively activated AKT (CA-AKT) or the corresponding controls (Ctrl or si-NC) using Lipofectamine 2000 (Thermo Fisher Scientific). Three shRNAs for MAPK4, three siRNAs for AKT1 and AKT2 were tested. The siRNA sequences are listed in Table S3. Oligos with highest efficiency were used for subsequent experiments. To construct the MAPK4-overexpressing vector, a One Step Cloning Kit (Vazyme Biotech, Nanjing) was used according to the manufacturer's instructions. The coding sequence (CDS) of MAPK4 was amplified using a human genomic DNA extracted from $\mathrm{SiHa}$ cells and then inserted into the PCI plasmid (Promega, Madison, WI, USA). Primers sequences for cloning MAPK4 CDS into the expressing vector are listed in Table S4. CA-AKT plasmid was purchased from Addgene (catalog no. plasmid \#14751, Watertown, MA).

\section{Synergistic effect of MAPK4 deletion with PARP1 inhibitors}

After cells were incubated with different concentrations of PARP1 inhibitors, olaparib or veliparib, cell viablity was determined by a cell counting kit-8 (CCK-8, Dojindo, Tokyo, Japan). A microplate spectrometer (Thermo Fisher Scientific) was used to determine the absorbance (OD $=450$ $\mathrm{nm}$ ). Next, the $50 \%$ inhibitory concentration (IC50) was quantified using the sigmoidal dose-response function of GraphPad Prism. In addition, after knocking out MAPK4, $\mathrm{SiHa}$ or caSki cells were over-expressed with MAPK4 or CA-AKT. Accordingly, the IC50 was quantified.

\section{Xenograft mouse model}

Animal experiments were performed according to the Guidelines for the Care and Use of Laboratory Animals published by the National Institutes of Health [21] and approved by the Ethics Committee of Henan Cancer Hospital. Thymic BALB/c nude mice (female, four-six weeks, 16-18 g) were housed in a pathogen-free facility and used for xenograft studies. SiHa or caSki cells with MAPK4 deletion $\left(1 \times 10^{6}\right)$ and the control cells were subcutaneously injected into the right flanks of mice. When the tumors reached a diameter of 4-5 mm, mice were randomly assigned to different groups $(6$ mice/ group). Tumors were monitored and treated with $5 \mathrm{~Gy}$ irradiation every 2 days after the injection. The length (L) and width (W) of tumors were determined every week. Then, the volume of each tumor was calculated using the formula $\mathrm{V}=1 / 2 \mathrm{LW}^{2}$. At 4 weeks post-implantation, tumors were dissected from the mice. Protein expression of $\mathrm{p}-\mathrm{AKT}$, AKT, p-DNA-PK, MAPK and $\gamma \mathrm{H} 2 \mathrm{AX}$ in tumor tissues was analyzed in each group established by caSki cells. To study the synergistic effect of MAPK4 deletion with PARP1 inhibitors in vivo, olaparib $(100 \mathrm{mg} / \mathrm{kg})$ was administered by oral gavage once daily, in addition to irradiation treatment. Tumor volume $\left(\mathrm{mm}^{3}\right)$ of each group median was graphed over time to monitor tumor growth. 4 weeks later, tumors were harvested and photographed.

\section{Statistical analysis}

The statistical data were obtained from three independent experiments and fit the normal distribution. Data are presented as the mean \pm standard error of the mean (SEM). The investigator was blinded to the group allocation during the experiment. Statistical differences were analyzed using the Student $t$ test for two groups and ANOVA for multiple groups. Variation within each group of data was estimated, and the variance between groups was statistically compared. $P<0.05$ was considered as significant.

\section{Results \\ MAPK4 level is associated with the survival of cervical cancer patients}

To study the correlation between MAPK4 level and cervical cancer development, we determined the MAPK4
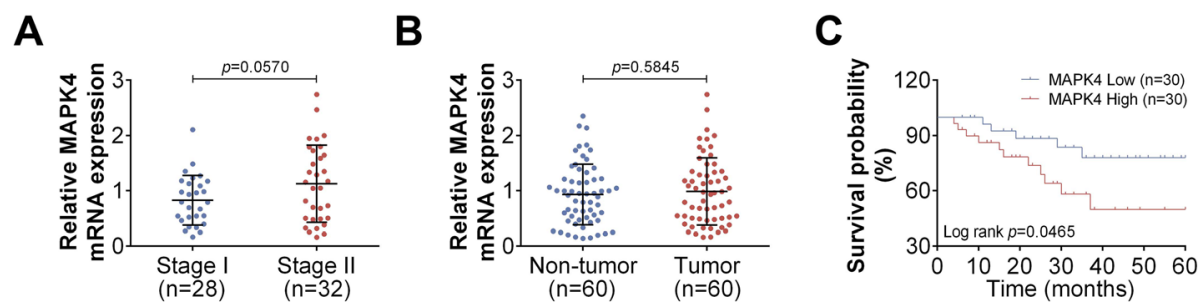

Fig. 1 MAPK4 mRNA level is associated with the overall survival of cervical cancer patients. a Relative MAPK4 mRNA levels in tissues of cervical cancer patients at stage I $(n=28)$ or stage II $(n=32)$, as determined using qRT-PCR. b Relative MAPK4 mRNA levels in cervical cancer tissues (tumor, $n=60$ ) and normal adjacent tissues (non-tumor, $n=60$ ), as determined using qRT-PCR. c Overall survival curve analysis of 60 cervical cancer patients using Kaplan-Meier method, stratified by MAPK4 mRNA expression. Data are expressed as the mean \pm SEM 
mRNA levels in cervical cancer tissues of different disease stages $(n=60)$. There was no significant difference in the relative MAPK4 mRNA level in stage II cervical cancer patients $(n=32)$ and stage I cervical cancer patients $(n=28$, Fig. 1a). Similar results were observed in tumor tissues (cervical cancer tissues, $n=60$ ) and non-tumor tissues (corresponding normal adjacent tumor tissues, $n=60$, Fig. 1b). However, when analyzing the relationships between MAPK4 mRNA level and the survival of cervical cancer patients after chemoradiotherapy, it was found that the survival rate of cervical cancer patients in "MAPK4 High" group was lower than that of patients in "MAPK4 Low" group (Fig. 1c). Because MAPK4 expression is associated with patient survival, these result suggest that MAPK4 may serve as a potential synergetic therapeutic target with PARP1 inhibitors in radiotherapy of cervical cancer. These findings led us to examine whether MAPK4 deletion can improve the effect of chemoradiotherapy for cervical cancer.

\section{MAPK4 knockout increases the sensitivity of cervical cancer cells to radiation}

To study the effect of MAPK4 knockout on the sensitivity of cervical cancer cells to radiation, MAPK4-deleted $\mathrm{SiHa}$ or caSki stable cell lines were established by CRIS PR-Cas9 technique. The western blotting results did not detect MAPK4 expression in the cells with MAPK4 knockout (MAPK4 KO), confirming the successful establishment of MAPK4 knockout cell line (Fig. 2a). Colony formation assay was then performed after radiation treatment. The results showed that MAPK4 knockout itself did not significantly affect the colony formation of cervical cancer cells (Fig. 2b). With radiation treatment at $5 \mathrm{~Gy}$, the colony number of MAPK4 knockout cells was markedly reduced compared to that of wild type (WT) cells. After MAPK4-knockout cervical cancer cells were treated with $5 \mathrm{~Gy}$ irradiation, the markers for DNA double-chain breakage were detected by immunofluorescence. After MAPK4 was knocked out, 53BP1 and $\gamma \mathrm{H} 2 \mathrm{AX}$ levels were significantly increased, indicating more severe DNA damage (Fig. 2c). Western blotting was used to assess the levels of proteins associated with
A
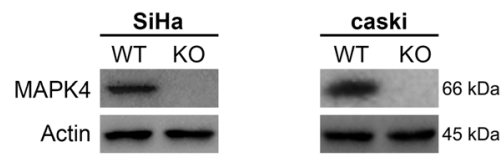

B
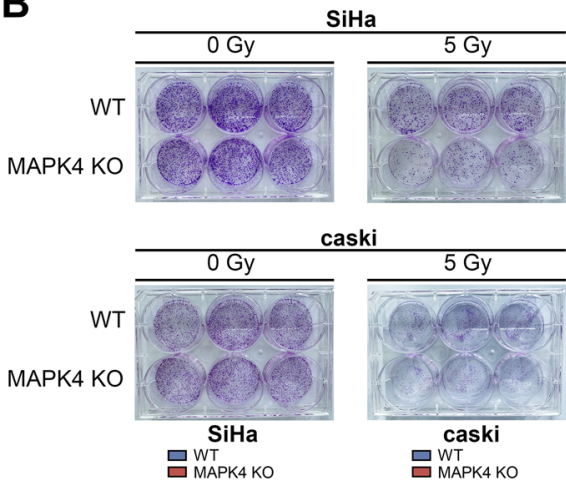

caski
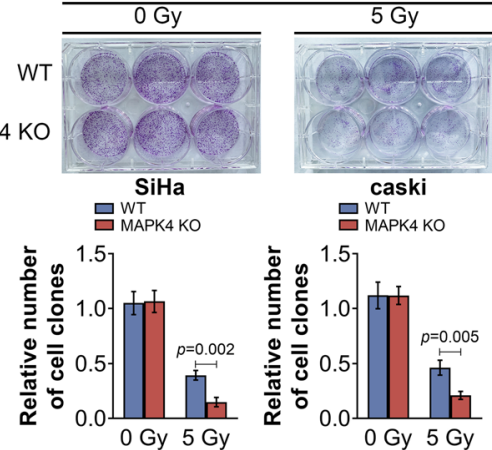

C

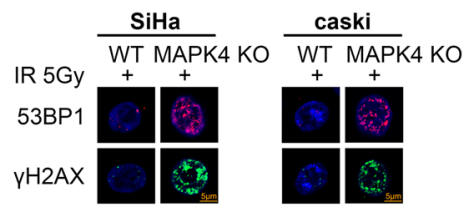

D

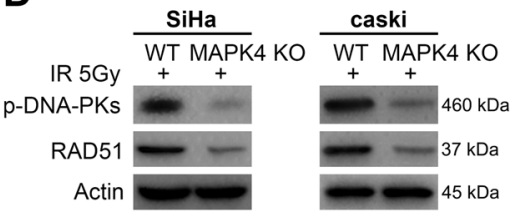

E

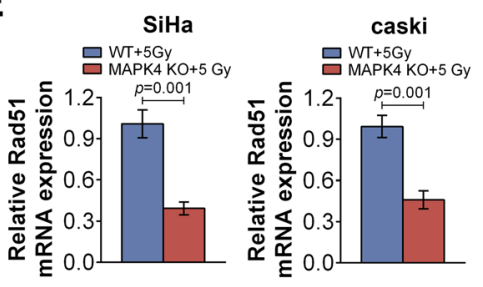

Fig. 2 MAPK4 knockout increases the sensitivity of cervical cancer cells to radiation. a Relative protein levels of MAPK4 in SiHa or caSki WT cells and MAPK $4 \mathrm{KO}$ cells after radiation treatment, as determined using western blotting. $\mathbf{b}$ Colony formation analysis of SiHa or caSki WT cells and MAPK4 KO cells after radiation treatment. The number of cell clones was quantified and shown as histogram. c The 53BP1 and $\gamma \mathrm{H} 2 \mathrm{AX}$ levels in SiHa or caSki WT cells and MAPK4 KO cells after radiation treatment, as determined using immunofluorescence. Representative images of three independent experiments are shown (Scale bar, $5 \mu \mathrm{m}$ ). d Relative protein levels of p-DNA-PK and RAD51 in SiHa or caSki WT cells and MAPK4 KO cells after radiation treatment, as determined using western blotting. e Relative RAD51 mRNA levels in SiHa or caSki WT cells and MAPK4 KO cells after radiation treatment, as determined using $\mathrm{qRT}-\mathrm{PCR}$. Data are expressed as the mean \pm SEM 


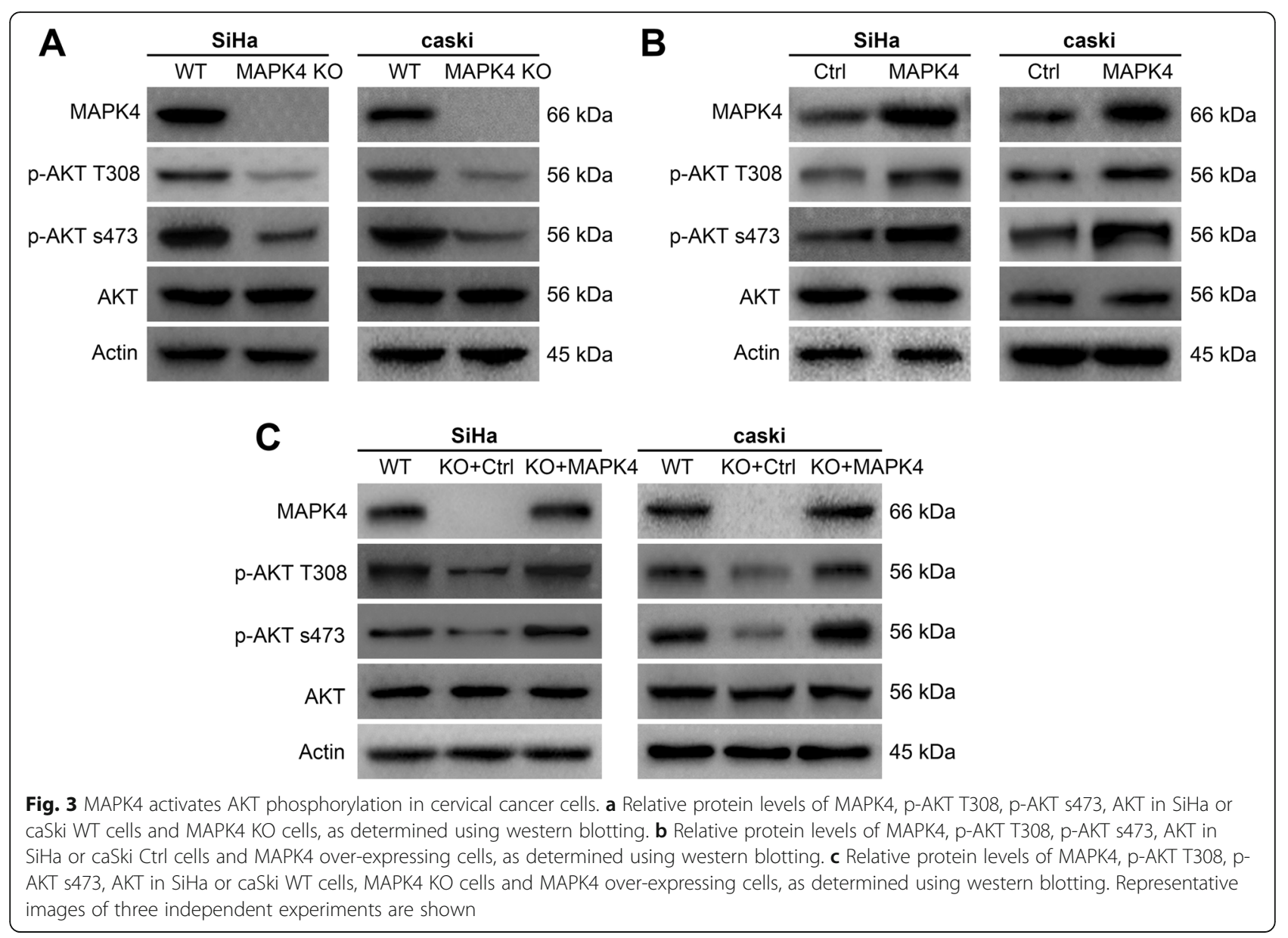

DNA repair. p-DNA-PK and RAD51 were significantly decreased following MAPK4 knockout, indicating that DNA repair was weakened (Fig. 2d). This was further confirmed by qRT-PCR results, which showed that the transcript levels of RAD51 in SiHa and caSki cells were significantly decreased (Fig. 2e). Together, these data suggest that MAPK4 knockout could down-regulate cell proliferation, induce DNA damage and attenuate DNA repair in cervical cancer cells after irradiation treatment.

\section{MAPK4 activates AKT phosphorylation in cervical cancer cells}

As shown in Fig. 3a, western blotting was used to determine the protein levels of p-AKT T308, p-AKT s473 and AKT in MAPK4-knockout SiHa or caSki cells. The absence of MAPK4 led to a decrease in the phosphorylation levels of AKT at T308 and s473 sites, but the total amount of AKT remained unaltered, suggesting that MAPK4 knockdown may inhibit AKT phosphorylation. In addition, the phosphorylation levels of AKT were determined in MAPK4-overexpressing cervical cancer cells, which demonstrated an increase in phosphorylated
AKT, but had no significant change in the levels of total AKT. This suggests that MAPK4 overexpression can promote AKT phosphorylation (Fig. 3b). Next, we further explored whether dysregulated AKT phosphorylation was directly caused by MAPK4 knockout or over-expression. We re-expressed MAPK4 using specific expressing plasmids in MAPK4 knockout cervical cancer cells, and examined the phosphorylation levels of AKT. Our results showed a decrease in phosphorylated AKT following MAPK4 knockout, which was restored by over-expressing MAPK4. There was no significant change in total AKT (Fig. 3c). Thus, these data further confirmed that MAPK4 could promote AKT phosphorylation.

\section{MAPK4 affects DNA repair through regulating AKT phosphorylation}

As mentioned above, we found that MAPK4 could affect DNA repair and the phosphorylation of AKT. Next, we sought to determine whether MAPK4 affects DNA repair through regulating AKT phosphorylation. Cervical cancer cells were transfected with AKT1 or AKT2 siRNA and subjected to irradiation treatment. The western blotting 


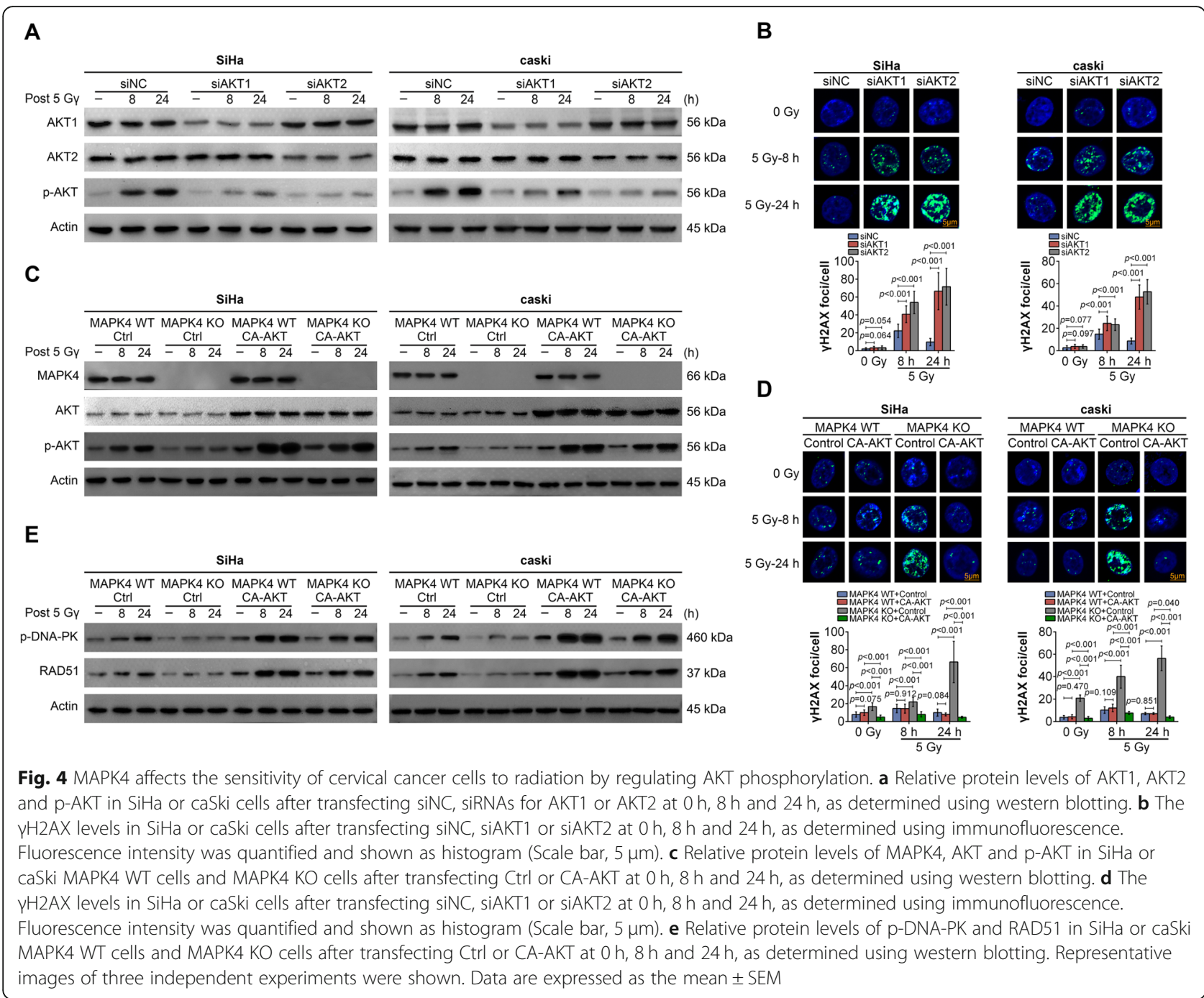

results showed that after AKT1 and AKT2 were knocked down, AKT1, AKT2 and p-AKT levels were decreased. Irradiation treatment led to the up-regulation of $\mathrm{p}$-AKT. However, when AKT1 or AKT2 was silenced, the effect of irradiation treatment on the p-AKT level was reduced (Fig. 4a). Accordingly, immunofluorescence results showed that $\gamma \mathrm{H} 2 \mathrm{AX}$ levels were increased after AKT1 or AKT2 was knocked down, indicating that AKT reduction could promote DNA damage (Fig. 4b). In addition, p-AKT and $\gamma \mathrm{H} 2 \mathrm{AX}$ levels were determined in MAPK4-knockout cervical cancer cells that constitutively expressed AKT. The over-expression of CA-AKT led to an increased pAKT level (Fig. 4c) and a reduction in $\gamma \mathrm{H} 2 \mathrm{AX}$ (Fig. 4d) in MAPK4 KO cells with irradiation treatment. Besides, the levels of DNA repair related proteins, $\mathrm{p}$-DNA-PK and RAD51, were determined in MAPK4 KO cells with irradiation treatment and CA-AKT over-expression. After overexpressing CA-AKT, p-DNA-PK and RAD51 levels were significantly up-regulated, suggesting that CA-AKT could promote DNA repair (Fig. 4e). These data above revealed that MAPK4 affected DNA repair through regulating AKT phosphorylation in cervical cancer cells.

\section{MAPK4 knockout enhances the sensitivity of cervical cancer cells to PARP1 inhibitors}

Given that MAPK4 knockout led to defective repair of DNA double-strand breaks, we next speculated that MAPK4 knockout and PARP1 inhibition may exert synergistic effects, leading to dysfunctional DNA-SSB repair. Thus, we then investigated the effect of MAPK4 knockout on the sensitivity of cervical cancer cells to PARP1 inhibitors, olaparib or veliparib. As shown in Fig. 5a, the IC50 value of olaparib in $\mathrm{SiHa}$ or caSki cells was significantly decreased after MAPK4 knockout. Similar results were observed in veliparib treated-SiHa or caSki cells. Furthermore, the IC50 of PARP1 inhibitors was detected by re-expressing MAPK4 or overexpressing CA-AKT in MAPK4 knockout cervical cancer cells. As shown in Fig. 5b, decreased-IC50 in MAPK4 KO 


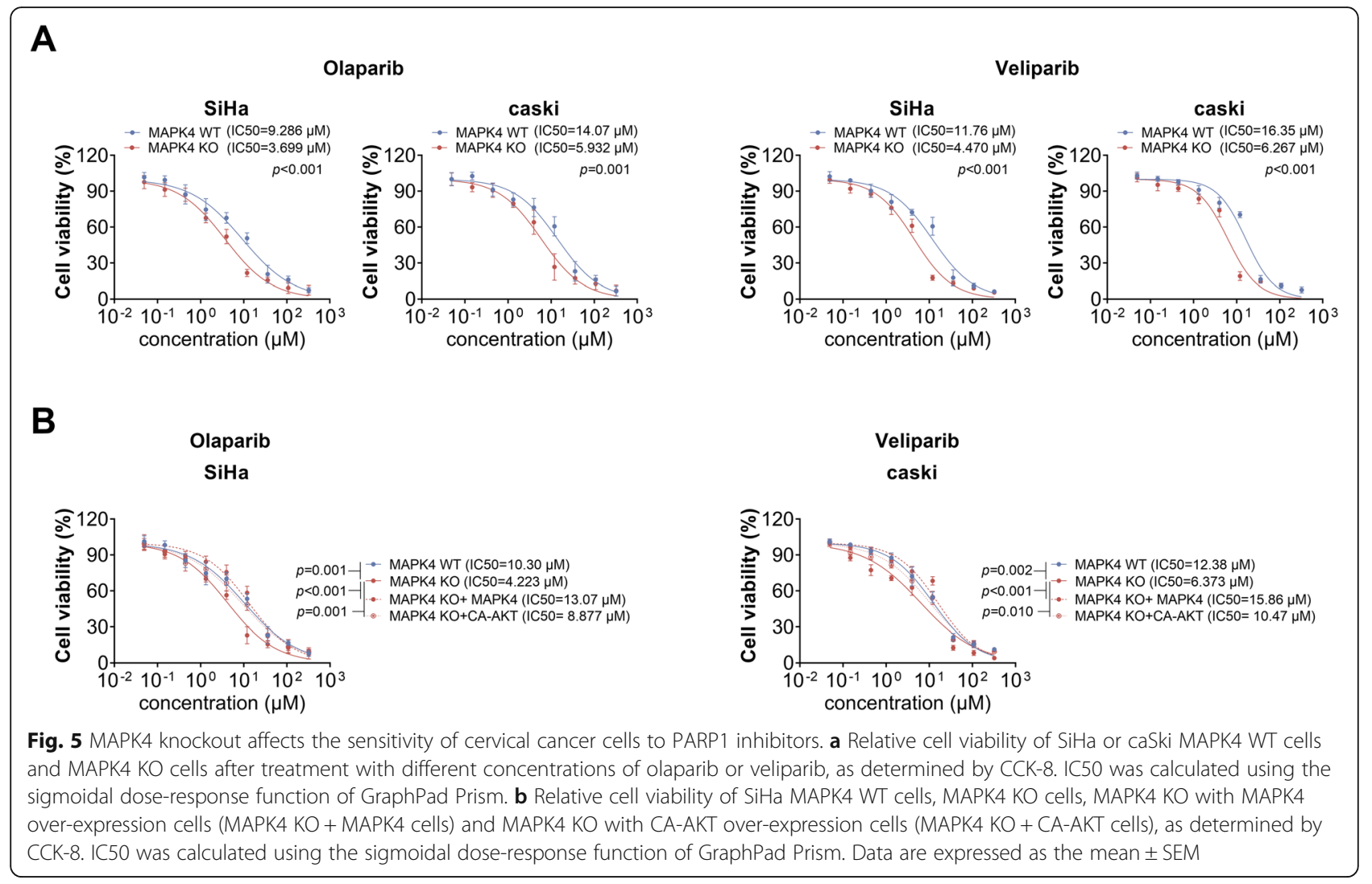

cells could be restored after re-expressing MAPK4 or overexpressing CA-AKT. These findings suggest that MAPK4 affects the sensitivity of cervical cancer cells to PARP1 inhibitors, through activating AKT phosphorylation.

\section{MAPK4 knockout enhances the sensitivity of cervical cancer to radiation and PARP1 inhibitors in vivo}

Given that MAPK4 deletion enhanced the sensitivity of cervical cancer cells to radiation and PARP1 inhibitors, we next investigated the effect of MAPK4 deletion in vivo. To construct a xenograft tumor mouse model, MAPK4-deleted stable SiHa and caSki cell lines were subcutaneously injected into nude mice. Subsequently, irradiation treatment was performed once a day at $2 \mathrm{~Gy}$, and the tumor growth curve was detected. As shown in Fig. 6a, MAPK4 knockout inhibited tumor growth to a certain extent. This observation was more evident after irradiation, in which MAPK4 knockout caused a more significant inhibition on tumor development. Furthermore, p-AKT, AKT, p-DNA-PK, MAPK4 and $\gamma \mathrm{H} 2 \mathrm{AX}$ protein levels were determined in xenograft tumors (Fig. 6b). MAPK4 knockout, especially after irradiation, led to downregulated p-AKT and pDNA-PK and up-regulated $\gamma \mathrm{H} 2 \mathrm{AX}$. In addition, after MAPK4 $\mathrm{KO}$ cells were subcutaneously injected into the mice, gavage of PARP1 inhibitor olaparib was performed irradiation treatment once a day. The results suggest a synergistic inhibitory effect of MAPK4 knockout and PARP1 inhibitor on tumor growth in vivo (Fig. 6c). Together, these findings demonstrated that MAPK4 knockout could inhibit DNA repair and improve the sensitivity of cervical cancer to irradiation treatment and PARP1 inhibitors in vivo.

\section{Discussion}

In this study, we have not only demonstrated the suppressive role of MAPK4 knockout on AKT phosphorylation, but also identified the relationship between MAPK4 knockout and enhanced sensitivity of cervical cancer to radiation treatment and PARP1 inhibitors in cells and mouse models. Although intensity-modulated radiotherapy (IMRT) has been adopted to reduce gastrointestinal toxicity and increase the radiotherapy dose, treatment tolerance and severe side effects are dose-limiting factors. Thus, novel developments aim to improve radiotherapy for cervical cancer [22]. It was demonstrated that concomitant radiochemotherapy could improve disease-free and overall survival compared to radiotherapy alone in early cervical cancer [23]. For example, methyl jasmonate (MJ), a newly identified cytotoxic agent, when effectively incorporated with X-ray irradiation, can significantly reduce the radiation doses required to inhibit cell survival of cervical cancer cells [24]. Additionally, the combination of Aloe-emodin (AE), an Aloe vera leaf exudate and radiation induce apoptosis and further improve Alkaline 


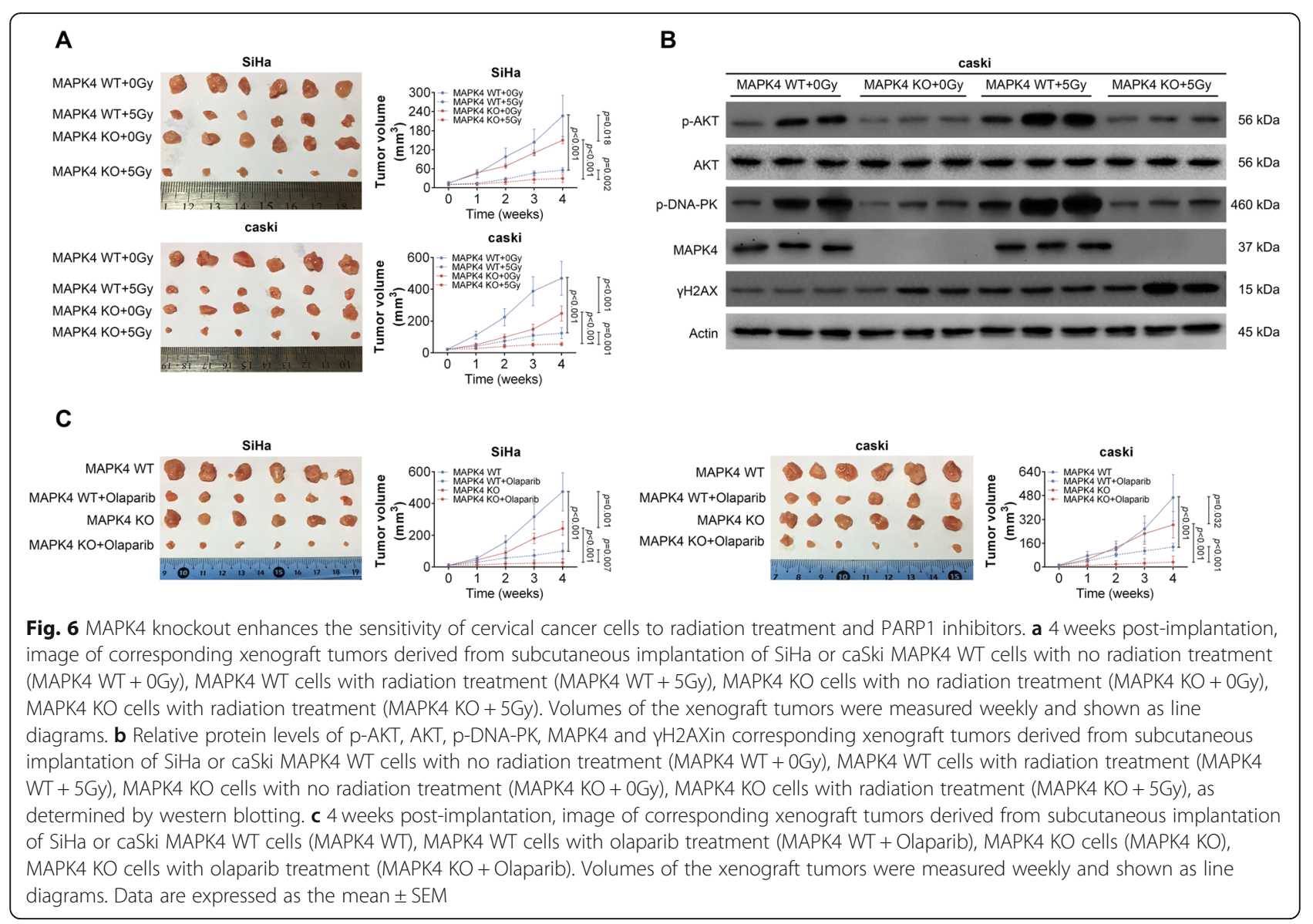

phosphatase (ALP) activity compared with treatment with $\mathrm{AE}$ or radiation alone [25]. Our data in this study demonstrated that MAPK4 knockout could enhance the sensitivity of cervical cancer cells to radiation treatment both in vitro and in vivo, suggesting that targeting MAPK4 may be a promising radiosensitizer.

As an atypical member of the mitogen-activated protein (MAP) kinase family, MAPK4 knockout mice are viable and fertile and exhibit no gross morphological or physiological anomalies. However, MAPK4-deficient mice manifest depression-like behavior in forced-swimming tests, indicating that the MAPK4 has acquired specialized functions through evolutionary diversification [26]. So far, little is known about the physiological function of MAPK4 and its involvement in diseases, including cancer. Although gene expression profiling data provided by The Cancer Genome Atlas (TCGA) show that MAPK4 expression is correlated with the survival rates in patients with lung cancer, bladder cancer and glioma, its functions and mechanism of actions in lung cancer and colon cancer were recently identified [13]. Wang et al. demonstrated that over-expression of MAPK4 leads to oncogenic effects, and MAPK4 inhibition suppresses cell proliferation and xenograft tumor growth. Mechanistically, MAPK4 activates the phosphorylation of AKT at threonine 308 and serine 473 [14]. Our data in this study demonstrated that cervical cancer patients with high MAPK4 expression had lower survival probability and MAPK4 deletion blocked AKT phosphorylation in cervical cancer cells. AKT phosphorylation has previously been described to cooperate with DNA-PKcs and was involved in DNA damage repair. AKT1 is a regulatory component in the homologous recombination repair of DNA-DSB in a Rad51-dependent manner in non-small cell lung cancer cells [27]. Single knockdown of Akt1 and Akt2 leads to a decrease in Rad51 foci formation and significantly reduces Rad51 protein level in colon cancer cells [28]. Moreover, Akt1-T308A/S473Aexpressing cells are characterized by increased radiosensitivity compared to Akt1-WT (wild type)-expressing cells in long-term colony formation assays [29]. Dual targeting of mTORC1 and AKT1 inhibits DNA-DSB repair, leading to radiosensitization of solid tumor cells [30]. We found that MAPK4-knockout cervical cancer cells showed lower AKT phosphorylation level, and had heightened sensitivity to radiation treatment and PARP1 inhibitors. 
In regard to the upstream regulation of MAPK4, two miRNAs have been reported to specifically target MAPK4. Over-expression of miR-767-5p functions as a tumor drive through targeting MAPK4 in multiple myeloma [31]. miR-127 was found to target both MAPK4 and HOXC6, and promotes cell proliferation and decreases differentiation in porcine [32]. These indicate that the expression and functions of MAPK4 may vary depending on the cellular context. To date, the regulatory mechanism of MAPK4 in cervical cancer remains unclear, and whether or not miR-767-5p and miR-127 could target MAPK4 and other potential transcriptional regulatory factors will require further investigation.

Because radiotherapy alone or concurrent chemoradiation fail to control advanced cervical cancer, surgery, chemotherapy or targeted therapy have been used in combination to improve the radiotherapy and minimize the side effects. Studies are currently being carried out to investigate potential suppressors of survival pathways and promoters of apoptotic pathways as novel chemotherapy approaches for the treatment of cervical cancer [33]. An association between poly ADP-ribose polymerase 1 (PARP1) Val762Ala polymorphism (rs1136410) and cancer therapy response has been identified, and PARP1 genotypes have been proposed to be an independent prognostic factor in cervical cancer [34]. In addition, it has been shown that PARP-1 inhibition may augment cisplatin cytotoxicity in cervical cancer cells by diminishing DNA repair and suppressing $\beta$-catenin signaling pathway [35]. miR-7-5p was demonstrated to negatively regulate PARP-1 protein and gene expression in cisplatin-resistant cervical cancer cells, and facilitates DNA repair and maintain cell survival [36]. However, little is known about the therapeutic efficacy of PARP inhibitors in the treatment of cervical cancer, either as a single agent or in combination with MAPK4 knockout. PARP1 inhibition along with superoxide dismutase 1 (SOD1) inhibition could promote the synthetic lethal killing of RAD54B-deficient colorectal cancer cells [37]. Previous study has also shown that simultaneous treatment with PARP and RAD52 inhibitors exerts dual synthetic lethality in BRCA-deficient tumors [38]. Currently, PARP inhibitors are under clinical trials for BRCA1/ BRCA2-deficient breast cancer and ovarian cancer by the approach of synthetic lethal $[39,40]$. Our results demonstrate for the first time that addition of PARP inhibitor may improve therapeutic outcome of MAPK4-deficient cervical cancer treated with radiation.

\section{Conclusions}

Altogether, our data revealed that cervical cancer patients with high MAPK4 mRNA expression have lower survival rate. After radiation treatment, the colony number of MAPK4 knockout cells was markedly reduced, and the markers for DNA double-chain breakage were significantly up-regulated. In addition, MAPK4 knockout reduced the phosphorylation of AKT, whereas its over-expression exerted opposite effects. In MAPK4 KO cells with irradiation treatment, inhibition of AKT phosphorylation promoted DNA double-chain breakage, and constitutively activated AKT (CA-AKT) increased the levels of p-AKT and DNA repair related proteins, p-DNA-PK and RAD51. MAPK4 is further found to affect the sensitivity of cervical cancer cells to PARP1 inhibitors by activating AKT phosphorylation. Moreover, MAPK4 knockout enhanced the sensitivity of cervical cancer to radiation and PARP1 inhibitors in mouse xenograft models. Collectively, our data suggest that combined application of MAPK4 knockout and radiation treatment or PARP1 inhibition can be used as therapeutic strategy for advanced cervical carcinoma.

\section{Supplementary information}

Supplementary information accompanies this paper at https://doi.org/10. 1186/s13046-020-01644-5.

Additional file 1: Table S1. The primers for qRT-PCR assay. Table S2. Antibodies used in this study. Table S3. The sequences for siRNAs. Table S4. The primers for MAPK4 expressing plasmid construct.

Additional file 2. The prognosis of survival.

\section{Abbreviations}

MAPK4: Mitogen-activated protein kinase 4; IC50: Inhibitory concentration; CA-AKT: Constitutively activated AKT; p-AKT: Phosphorylated-AKT; p-DNAPK: Phosphorylated-DNA-dependent protein kinase; RAD51: RAD51 recombinase; PARP1: Poly ADP-ribose polymerase 1; HPV: Hhuman papillomavirus; NCCN: National Comprehensive Cancer Network; MAPKK: MAPK kinase; TCGA: The Cancer Genome Atlas; AKT: Activates protein kinase B; PI3K: Phosphatidylinositol 3-hydroxykinase;

PARP1: Polymerase 1

\section{Acknowledgements}

Not applicable.

\section{Authors' contributions}

SZT and LLL conceived and designed the experiments, MYT and GPL analyzed and interpreted the results of the experiments, JHT and XC performed the experiments. The authors read and approved the final manuscript.

\section{Funding \\ Not applicable.}

\section{Availability of data and materials}

All data generated or analyzed during this study are included in this published article.

\section{Ethics approval and consent to participate}

All patients were pathologically and clinically diagnosed as cervical cancer and received no chemotherapy or radiotherapy before section. The collection and experiments were performed under the permission of the Ethics Committee of Henan Cancer Hospital (Approval no.2016HC047). Written informed consents were obtained from all participants.

Animals published by the National Institutes of Health and approved by the Ethics Committee of Henan Cancer Hospital.

Consent for publication

Not applicable. 


\section{Competing interests}

The authors state that there are no conflicts of interest to disclose.

\section{Author details}

'Department of Gynecology, Affiliated Cancer Hospital of Zhengzhou University, Henan Procincical Cancer Hospital, No. 127 Dongming Avenue, Zhengzhou City 450009, Henan Province, China. ${ }^{2}$ Department of Respiratory Medicine, The First Affiliated Hospital Zhengzhou University, Zhengzhou City 450052, Henan Province, China. ${ }^{3}$ Department of Pathology, Affiliated Cancer Hospital of Zhengzhou University, Henan Procincical Cancer Hospital, Zhengzhou City 450009, Henan Province, China. ${ }^{4}$ Department of Emergency Medicine, The First Affiliated Hospital Zhengzhou University, Zhengzhou City 450052, Henan Province, China. ${ }^{5}$ Department of Internal Medicine, Peking University Hospital, Beijing 100871, China. ${ }^{6}$ School of Basic Medicine, Zhejiang University Medical School, Hangzhou City 310013, Zhejiang Province, China.

Received: 25 May 2020 Accepted: 15 July 2020

Published online: 25 July 2020

\section{References}

1. Bray F, Ferlay J, Soerjomataram I, Siegel RL, Torre LA, Jemal A. Global cancer statistics 2018: GLOBOCAN estimates of incidence and mortality worldwide for 36 cancers in 185 countries. CA Cancer J Clin. 2018;68(6):394-424.

2. Marth C, Landoni F, Mahner S, McCormack M, Gonzalez-Martin A, Colombo $\mathrm{N}$, et al. Cervical cancer: ESMO Clinical Practice Guidelines for diagnosis, treatment and follow-up. Ann Oncol. 2018;29(Suppl 4):iv262.

3. Tse $\mathrm{K}, \mathrm{Ngan} \mathrm{H}$. World trends of incidence of gynecologic malignancies in young women. Eur J Gynaecol Oncol. 2018;39(6):871-6.

4. Eiben GL, da Silva DM, Fausch SC, Le Poole IC, Nishimura MI, Kast WM. Cervical cancer vaccines: recent advances in HPV research. Viral Immunol. 2003;16(2):111-21.

5. Pimple SA, Mishra GA. Global strategies for cervical cancer prevention and screening. Minerva Ginecol. 2019;71(4):313-20.

6. Zhou J, Wu SG, Sun JY, Li FY, Lin HX, Chen QH, et al. Comparison of clinical outcomes of squamous cell carcinoma, adenocarcinoma, and adenosquamous carcinoma of the uterine cervix after definitive radiotherapy: a population-based analysis. J Cancer Res Clin Oncol. 2017; 143(1):115-22.

7. Chen JL, Huang CY, Huang YS, Chen RJ, Wang CW, Chen YH, et al. Differential clinical characteristics, treatment response and prognosis of locally advanced adenocarcinoma/adenosquamous carcinoma and squamous cell carcinoma of cervix treated with definitive radiotherapy. Acta Obstet Gynecol Scand. 2014;93(7):661-8.

8. Johnson GL, Lapadat R. Mitogen-activated protein kinase pathways mediated by ERK, JNK, and p38 protein kinases. Science. 2002;298(5600):1911-2.

9. Zhou G, Bao ZQ, Dixon JE. Components of a new human protein kinase signal transduction pathway. J Biol Chem. 1995;270(21):12665-9.

10. Gonzalez FA, Raden DL, Rigby MR, Davis RJ. Heterogeneous expression of four MAP kinase isoforms in human tissues. FEBS Lett. 1992;304(2-3):170-8.

11. Kant S, Schumacher S, Singh MK, Kispert A, Kotlyarov A, Gaestel M. Characterization of the atypical MAPK ERK4 and its activation of the MAPK-activated protein kinase MK5. J Biol Chem. 2006;281(46):35511-9.

12. Aberg E, Perander M, Johansen B, Julien C, Meloche S, Keyse SM, et al. Regulation of MAPK-activated protein kinase 5 activity and subcellular localization by the atypical MAPK ERK4/MAPK4. J Biol Chem. 2006;281(46):35499-510.

13. Cancer Genome Atlas Research N, Weinstein JN, Collisson EA, Mills GB, Shaw KR, Ozenberger BA, et al. The Cancer Genome Atlas pan-Cancer analysis project. Nat Genet. 2013;45(10):1113-20.

14. Wang W, Shen T, Dong B, Creighton CJ, Meng Y, Zhou W, et al. MAPK4 overexpression promotes tumor progression via noncanonical activation of AKT/mTOR signaling. J Clin Invest. 2019;129(3):1015-29.

15. Toulany M, Lee KJ, Fattah KR, Lin YF, Fehrenbacher B, Schaller M, et al. Akt promotes post-irradiation survival of human tumor cells through initiation, progression, and termination of DNA-PKcs-dependent DNA double-strand break repair. Mol Cancer Res. 2012;10(7):945-57.

16. Schreiber V, Dantzer F, Ame JC, de Murcia G. Poly (ADP-ribose): novel functions for an old molecule. Nat Rev Mol Cell Biol. 2006;7(7):517-28.
17. Bryant HE, Schultz N, Thomas HD, Parker KM, Flower D, Lopez E, et al. Specific killing of BRCA2-deficient tumours with inhibitors of poly (ADPribose) polymerase. Nature. 2005;434(7035):913-7.

18. Prasad CB, Prasad SB, Yadav SS, Pandey LK, Singh S, Pradhan S, et al. Olaparib modulates DNA repair efficiency, sensitizes cervical cancer cells to cisplatin and exhibits anti-metastatic property. Sci Rep. 2017;7(1):12876.

19. Bianchi A, Lopez S, Altwerger G, Bellone S, Bonazzoli E, Zammataro L, et al. PARP-1 activity (PAR) determines the sensitivity of cervical cancer to olaparib. Gynecol Oncol. 2019;155(1):144-50.

20. Zhu D, Sun C, Qian X. MST1 suppresses viability and promotes apoptosis of glioma cells via upregulating SIRT6 expression. J Integr Neurosci. 2019;18(2):117-26.

21. Kastenmayer RJ, Moore RM, Bright AL, Torres-Cruz R, Elkins WR. Select agent and toxin regulations: beyond the eighth edition of the guide for the care and use of laboratory animals. J Am Assoc Lab Anim Sci. 2012;51(3):333-8.

22. Vordermark D. Radiotherapy of cervical Cancer. Oncol Res Treat. 2016;39(9): 516-20.

23. Einhorn N, Trope C, Ridderheim M, Boman K, Sorbe B, Cavallin-Stahl E. A systematic overview of radiation therapy effects in cervical cancer (cervix uteri). Acta Oncol. 2003;42(5-6):546-56.

24. Milrot $E$, Jackman A, Flescher $E$, Gonen P, Kelson I, Keisari $Y$, et al. Enhanced killing of cervical cancer cells by combinations of methyl jasmonate with cisplatin, $X$ or alpha radiation. Investig New Drugs. 2013;31(2):333-44.

25. Luo J, Yuan Y, Chang P, Li D, Liu Z, Qu Y. Combination of aloe-emodin with radiation enhances radiation effects and improves differentiation in human cervical cancer cells. Mol Med Rep. 2014;10(2):731-6.

26. Rousseau J, Klinger S, Rachalski A, Turgeon B, Deleris P, Vigneault E, et al. Targeted inactivation of Mapk4 in mice reveals specific nonredundant functions of Erk3/Erk4 subfamily mitogen-activated protein kinases. Mol Cell Biol. 2010;30(24):5752-63.

27. Mueck K, Rebholz S, Harati MD, Rodemann HP, Toulany M. Akt1 Stimulates Homologous Recombination Repair of DNA Double-Strand Breaks in a Rad51-Dependent Manner. Int J Mol Sci. 2017;18(11):2473.

28. Mohammadian Gol T, Rodemann HP, Dittmann K. Depletion of Akt1 and Akt2 Impairs the Repair of Radiation-Induced DNA Double Strand Breaks via Homologous Recombination. Int J Mol Sci. 2019;20(24):6316.

29. Szymonowicz K, Oeck S, Krysztofiak A, van der Linden J, Iliakis G, Jendrossek V. Restraining Akt1 Phosphorylation Attenuates the Repair of RadiationInduced DNA Double-Strand Breaks and Reduces the Survival of Irradiated Cancer Cells. Int J Mol Sci. 2018;19(8):2233.

30. Holler M, Grottke A, Mueck K, Manes J, Jucker M, Rodemann HP, et al. Dual targeting of Akt and mTORC1 impairs repair of DNA double-Strand breaks and increases radiation sensitivity of human tumor cells. PLoS One. 2016; 11(5):e0154745.

31. Feng Y, Zhang L, Wu J, Khadka B, Fang Z, Gu J, et al. CircRNA circ_0000190 inhibits the progression of multiple myeloma through modulating miR-7675p/MAPK4 pathway. J Exp Clin Cancer Res. 2019;38(1):54

32. Gao Y, Wang Y, Chen X, Peng Y, Chen F, He Y, et al. MiR-127 attenuates adipogenesis by targeting MAPK4 and HOXC6 in porcine adipocytes. J Cell Physiol. 2019;234(12):21838-50.

33. Hougardy BM, Maduro JH, van der Zee AG, Willemse PH, de Jong S, de Vries EG. Clinical potential of inhibitors of survival pathways and activators of apoptotic pathways in treatment of cervical cancer: changing the apoptotic balance. Lancet Oncol. 2005;6(8):589-98.

34. Nogueira A, Assis J, Faustino I, Pereira D, Catarino R, Medeiros R. Base excision repair pathway: PARP1 genotypes as modulators of therapy response in cervical cancer patients. Biomarkers. 2017;22(1):70-6.

35. Mann M, Kumar S, Sharma A, Chauhan SS, Bhatla N, Kumar S, et al. PARP-1 inhibitor modulate beta-catenin signaling to enhance cisplatin sensitivity in cancer cervix. Oncotarget. 2019;10(42):4262-75.

36. Yang F, Guo L, Cao Y, Li S, Li J, Liu M. MicroRNA-7-5p promotes Cisplatin resistance of cervical Cancer cells and modulation of cellular energy homeostasis by regulating the expression of the PARP-1 and BCL2 genes. Med Sci Monit. 2018:24:6506-16.

37. McAndrew EN, Lepage CC, McManus KJ. The synthetic lethal killing of RAD54B-deficient colorectal cancer cells by PARP1 inhibition is enhanced with SOD1 inhibition. Oncotarget. 2016;7(52):87417-30.

38. Sullivan-Reed K, Bolton-Gillespie E, Dasgupta Y, Langer S, Siciliano M, Nieborowska-Skorska M, et al. Simultaneous targeting of PARP1 and RAD52 triggers dual synthetic lethality in BRCA-deficient tumor cells. Cell Rep. 2018;23(11):3127-36 
39. Tutt A, Robson M, Garber JE, Domchek SM, Audeh MW, Weitzel JN, et al. Oral poly (ADP-ribose) polymerase inhibitor olaparib in patients with BRCA1 or BRCA2 mutations and advanced breast cancer: a proof-of-concept trial. Lancet. 2010;376(9737):235-44.

40. Audeh MW, Carmichael J, Penson RT, Friedlander M, Powell B, Bell-McGuinn $\mathrm{KM}$, et al. Oral poly (ADP-ribose) polymerase inhibitor olaparib in patients with BRCA1 or BRCA2 mutations and recurrent ovarian cancer: a proof-ofconcept trial. Lancet. 2010;376(9737):245-51.

\section{Publisher's Note}

Springer Nature remains neutral with regard to jurisdictional claims in published maps and institutional affiliations.

Ready to submit your research? Choose BMC and benefit from:

- fast, convenient online submission

- thorough peer review by experienced researchers in your field

- rapid publication on acceptance

- support for research data, including large and complex data types

- gold Open Access which fosters wider collaboration and increased citations

- maximum visibility for your research: over $100 \mathrm{M}$ website views per year

At $\mathrm{BMC}$, research is always in progress.

Learn more biomedcentral.com/submissions 